\title{
Conical Beams for the Analysis of Beam Waveguides
}

\author{
M. Casaletti ${ }^{(1)}$, S. Skokic ${ }^{(2)}$, Stig Sørensen ${ }^{(3)}$, and S. Maci ${ }^{(1)}$ \\ (1) Department of Information Engineering, University of Siena, Italy \\ (2) Faculty of Electrical Engineering and Computing, Zagreb, Croatia \\ (3) TICRA, Copenhagen, Denmark \\ E-mail: sinisa.skokic@fer.hr, casaletti@dii.unisi.it, macis@dii.unisi.it, \\ sbs@ticra.com
}

\begin{abstract}
This paper discusses a novel method for computing propagation and reflection of beams in a beam waveguide by combining a new type of wave-objects with Physical Optics. The wave objects are generated starting from the electric field spectrum in the focal plane, by applying the Fourier Series and the Generalised Pencil-of-Function expansion. This transforms the radiation integral to a form which rigorously respects the wave equation and can be evaluated in analytical closed form. The wave objects are launched to the reflector and reflected to the subsequent focal plane via Physical Optics. By computing the Fourier spectrum in the new focal plane it is possible to re-expand the reflected field into a new set of wave-objects, which is the foundation of beam waveguide analysis.
\end{abstract}

\section{Introduction}

The analysis of reflector systems at mm-wave frequencies requires the development of new analysis methods particularly suited for that frequency and size range. For example, a beam waveguide can be composed of a sequence of reflectors, each of which can be a hundred wavelengths in diameter. Hence, instead of discretising the analysis domain like classical numerical methods do (e.g. Method of Moments or even Physical Optics), the methods developed for this area express the field in terms of a relatively low number of beams. Beams are, generally speaking, wave-objects of higher complexity whose propagation, reflection, and diffraction if possible, can be resolved at least asymptotically in a closed form. The aim is to develop a modular process, whereby the total reflected field can be re-expanded into a new sum of beams, and the whole procedure can be repeated, so as to enable the analysis of complex multi-reflector systems, e.g. the beam waveguide.

A number of different objects have been introduced that are more or less suitable to the exposed analysis approach. These are the Gaussian Beams [1, 2], higherorder Gauss-Laguerre or Gauss-Hermite modes [3, 4], Complex Source Points [5], etc. Recently, we presented a new kind of wave objects (WO's) [6], introducing a formulation that has potential advantages over other approaches in that it satisfies the following important aspects: 1) the WO's respect the wave 
equation in all space where they are valid, and 2) the generation of these WO's is done in efficient and natural way starting from the aperture field expansion. Moreover, the WO's possess analytical expressions in both spatial domain and the zero order WO coincides with an ordinary spherical wave field. All these properties make the WO promising in the description of multi-reflector systems, whose conventional $\mathrm{PO}$ analysis results sometimes prohibitively slow.

\section{Cylindrical Wave Beams}

The WO is defined through its Fourier Transform as

$$
W_{n}(\rho, \phi, \tilde{z})=e^{-j n \phi} \int_{0}^{\infty} \frac{e^{-j z \sqrt{k^{2}-k_{\rho}^{2}}}}{\sqrt{k^{2}-k_{\rho}^{2}}} \mathrm{~J}_{n}\left(\rho k_{\rho}\right) k_{\rho} d k_{\rho} .
$$

It has been demonstrated in [6], that (1) can be evaluated in a closed form in the space domain by simple recurrence formula for every index $n$. For $n=0$, the representation (1) coincides with the Sommerfeld representation, and the zeroorder wave-object is found as $W_{0}=j \exp (-j k r) / r$. A similar result is obtained for $n=1$. Bearing in mind the recurrence relation between the wave-objects, it can be shown that the WO's respect the wave equation for every $n$.

The WO's are generated automatically from the spectral-domain aperture field representation, which is expressed here in cylindrical coordinates for convenience:

$$
I(\rho, \phi, z)=\frac{1}{8 \pi^{2} j} \int_{0}^{\infty} \int_{0}^{\infty \pi} g\left(k_{\rho}, \alpha\right) e^{\left.-j k_{\rho} \infty \omega \infty \alpha-\phi\right)} \frac{e^{-j \sqrt{k^{2}-k_{\rho}^{2}}}}{\sqrt{k^{2}-k_{\rho}^{2}}} k_{\rho} d k_{\rho} d \alpha
$$

Here, $g\left(k_{\rho}, \alpha\right)$ is the spectrum of any of the Cartesian components of the electric (or magnetic) field. The process of beam generation is done in two steps. First, $g\left(k_{\rho}, \alpha\right)$ is expanded via FFT in a Fourier series in the angular coordinate: $g\left(k_{\rho}, \alpha\right)=\sum_{n=-\infty}^{\infty} c_{n}\left(k_{\rho}\right) e^{-j n \alpha}$; next, the Generalized Pencil of Functions (GPoF) expansion is applied to the Fourier coefficients: $c_{n}\left(k_{\rho}\right)=\sum_{m=-M}^{M} d_{m n} e^{b_{m} \sqrt{k^{2}-k_{\rho}^{2}}}$. The double integral reduces to this double summation:

$$
I=\sum_{n} c_{n} \sum_{m=-M}^{M} d_{m n} W_{n}\left(\rho, \phi, z+j b_{m n}\right),
$$

where $W_{n}(\rho, \phi, \tilde{z})$ is the $n$-th order wave object, as defined in (1), evaluated at the point $(\rho, \phi, \tilde{z})$ with a complex coordinate $\tilde{z}=z+j b_{m n}$. This means that the initial radiation integral has been reduced to a sum of functions that are well-behaved in the whole upper half-space $(\operatorname{Re}\{\tilde{z}\}>0)$. 


\section{Analysis of Reflection Systems Using Wave Objects}

In this paper the expansion in (3) is used for the analysis of the basic segment of a beam waveguide, constituted by a Gregorian beam-excited reflector (Figs. 1a).

The field around the focal point $F_{1}$ of an elliptical reflector is expanded in terms of wave-objects in the aperture focal plane. Each beam propagates independently to the first reflector of the chain, and Physical Optics (PO) is used for treating the its scattering by the reflector. The spectrum of the scattered field at the infinite dimension aperture of the second focal plane $F_{2}$ can be obtained from the scattered far field and used to re-expanded the field in terms of a new set of beams. The main advantage of this approach is that it requires only a few beams at each step, due to the angular selectivity property of wave-objects, which saves much computational time.

In order to analyse a multi-reflector beam waveguide, the process is to be repeated for every segment of the waveguide (Fig. 1b). The whole reflector system is then set up as a cascade of interconnected blocks with a low number of ports, corresponding to the number of wave-objects used in the analysis of each block
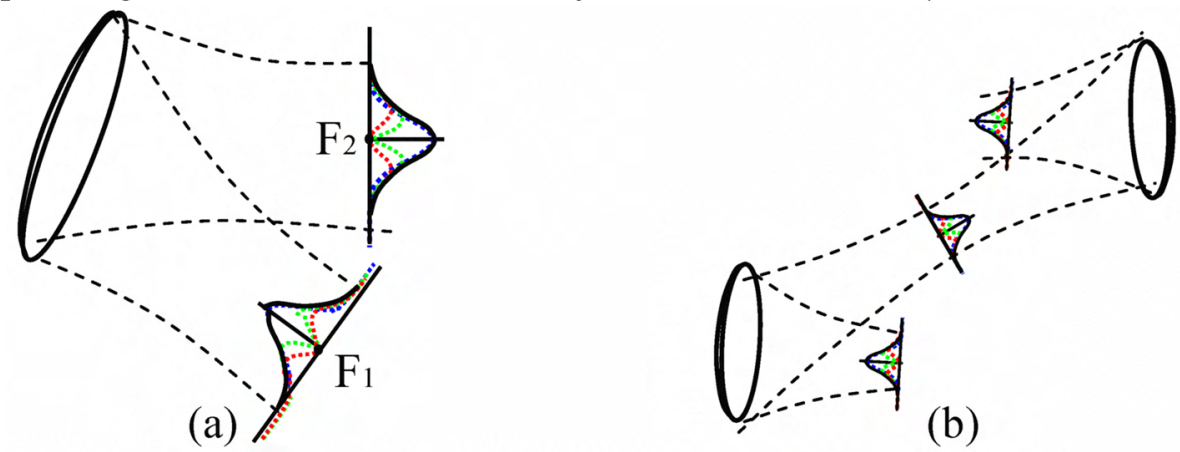

Fig. 1 (a) Basic segment of a Beam Waveguide. (b) Cascade of segments in a Beam Waveguide..

\section{Numerical Results}

As a preliminary example we will consider the analysis of a segment of a beam waveguide. The rim of the reflector is defined by the intersection of a ellipsoid surface (with axis $a=8 \lambda \quad b=4 \lambda$ ) with a cone of half opening angle of $15^{\circ}$ and apex in the focal point. The reflector is illuminated by a Gaussian feed with halfwidth beam $W=\lambda$ placed in the focal point. In fig. 2 is shown the radiated field on an half sphere located in the far-field region obtained by a standard PO method, in fig. 3 indeed is shown the radiated far field using the WO expansion presented in this paper. The results shown a good agreement between the two methods.

\section{References}

[1] N. J. McEwan and P. F. Goldsmith, "Gaussian Beam Techniques for Illuminating Reflector Antennas”, IEEE Trans. Antennas \& Propagation, vol. 37, No. 3, pp. 297304, 1989 
[2] H-T. Chou and P. H. Pathak, "Uniform Asymptotic Solution for Electromagnetic Reflection and Diffraction of an Arbitrary Gaussian Beam by a Smooth Surface with an Edge", Radio Science, vol. 32, no. 4, pp. 1319-1336, 1997.

[3] W.A. Imbriale and D.J. Hoppe, "Recent Trends in the Analysis of Quasioptical Systems", Proc. Millenium Conference on Antennas and Propagation, Davos, Switzerland, 2000.

[4] S. Withington, J.A. Murphy and K.G. Isaak, "Representation of Mirrors in Beam Waveguides as Inclined Phase-Transforming Surfaces", Infrared Phys. Technol., vol. 36, no. 3, pp. 723-734, 1995.

[5] Y. Dezhong, "Complex Source Representation of Time Harmonic Radiation from a Plane Aperture", IEEE Trans. Antennas \& Propagation, vol. 43, No. 7, pp. 720-723, 1995.

[6] S. Skokic, G.Carluccio, S.Maci, "New Wave-objects for Efficient Treatment of Complex Multi-reflector Systems", Proc. 2nd European Conference on Antennas and Propagation (EuCAP 2007), Edinburgh, United Kingdom, 2007
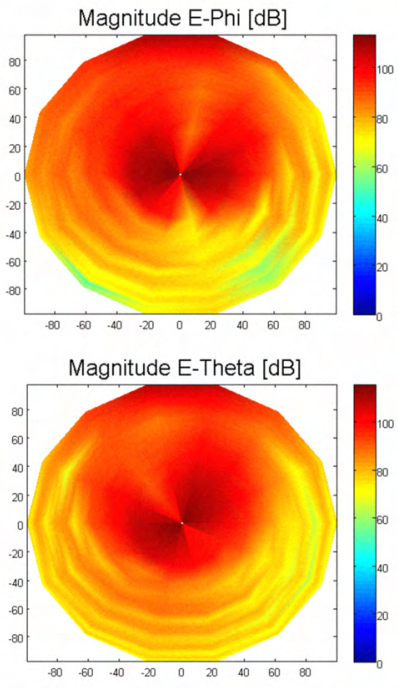

Fig.2 PO Radiated Field on a far field half-sphere
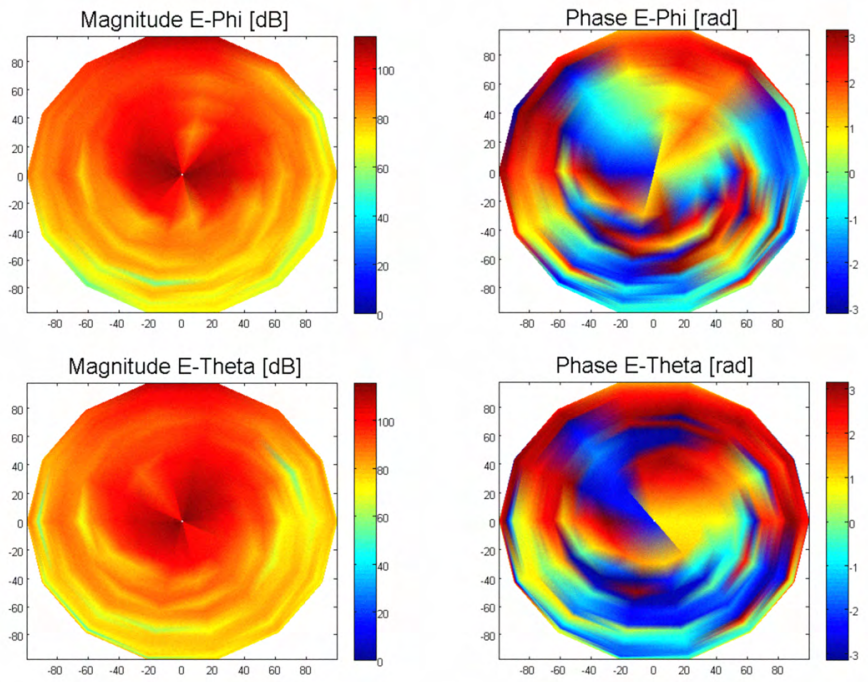

Fig.3 WO Radiated Field on a far field half-sphere 